\title{
Agent based modeling of energy networks
}

\author{
José María Gonzalez de Durana*, Oscar Barambones \\ University College of Engineering, University of the Basque Country, Nieves Cano 12, 01006 Vitoria-Gasteiz, Spain
}

Enrique Kremers

European Institute for Energy Research (Electricité de France 83 Karlsruhe Institute of Technology), Emmy-Noether-Strasse 11, 76131

Karlsruhe, Germany

Liz Varga

Complex Systems Research Centre, Cranfield School of Management, Cranfield University, Cranfield, Bedford MK43 OAL, U.K.

\begin{abstract}
Attempts to model any present or future power grid face a huge challenge because a power grid is a complex system, with feedback and multi-agent behaviors, integrated by generation, distribution, storage and consumption systems, using various control and automation computing systems to manage electricity flows.

Our approach to modeling is to build upon an established model of the low voltage electricity network which is tested and proven, by extending it to a generalised energy model.

But, in order to address the crucial issues of energy efficiency, additional processes like energy conversion and storage, and further energy carriers, such as gas, heat, etc., besides the traditional electrical one, must be considered. Therefore a more powerful model, provided with enhanced nodes or conversion points, able to deal with multidimensional flows, is being required.

This article addresses the issue of modeling a local multi-carrier energy network. This problem can be considered as an extension of modeling a low voltage distribution network located at some urban or rural geographic area. But instead of using an external power flow analysis package to do the power flow calculations, as used in electric networks, in this work we integrate a multiagent algorithm to perform the task, in a concurrent way to the other simulation tasks, and not only for the electric fluid but also for a number of additional energy carriers. As the model is mainly focused in system operation, generation and load models are not developed.
\end{abstract}

Keywords: complex systems, agent based modeling, electrical grid, smart grid, renewable energy systems, microgrids modeling, multi-carrier energy systems.

\section{Introduction}

It is an indisputable fact that levels of welfare, health and life expectancy that distinguish our present civilization are due to the progressive development of technology and the increasing use of energy. But it is clearly not possible to follow a development model based on continuous growth since, because our world is finite, there comes a time, sooner or later, in which the phenomenon called saturation appears: the finite resources are exhausted or reach intolerable levels.

After the first warnings on climate change were observed about 40 years ago, a number of scientists, experts and

\footnotetext{
* Corresponding author

Email addresses: josemaria.gonzalezdedurana@ehu.es (José María Gonzalez de Durana), oscar.barambones@ehu.es (Oscar Barambones), kremers@eifer.org (Enrique Kremers), liz.varga@cranfield.ac.uk (Liz Varga)
}

organizations began to do research and to publish alarming results on probable climate change. That number has grown steadily and today almost all nations of the world have scientists, experts and institutions dedicated to the study of the subject and promote initiatives to mitigate their effects, so there are thousands of organizations across the world devoted to climate studies, effects and corrective actions $[1,2,3]$.

\subsection{Energy systems research}

Some of the most important guidelines arising from practically all directives, policies and rules, with special significance to us are: increasing the use of renewable energy and improving efficiencies in generation, consumption and storage of energy.

Of special interest to our work are protection, monitoring and control systems at all voltage levels, equipment aiming at two-way digital communication, intelligent mon- 
itoring and management of electricity generation, transmission, distribution and consumption.

In addition to distributed electricity generation, the recommendations are for diversification of energy sources without relying too heavily on a single source of energy. So they are supporting the use of natural gas and cogeneration systems, cooperating with the electric network leading to a new energy network [2].

\subsection{Related work and scope}

A number of special software tools exist for simulation of electrical and hybrid systems, like HOMER, RETScreen, RAPSIM, Hybrid2, ViPOR, etc. However, although they allow the user for evaluating and optimising designs, using their built-in load models procedures and specialised procedures, all of them are closed systems that must be used as they are.

Regarding agent-based methodology, others have used it for energy network modeling. However, some applications are closely related to a particular technology, such as for example sensors and communication protocols [4] and others try to model very special problems, such as for example outages and faults in the power grid [5]. Even the idea of the creation of an agent based modeling of energy networks has been published in other papers [6], but mainly focused on optimization problems.

The original contribution presented in this paper consists of setting up a general approach for modeling multicarrier energy systems, in a completely open and selfcontained way, only based in the mathematical equations of energy networks, and therefore independent of any technology, with ability to model any energy system, from small systems (as for example the power network in an electric car), to bigger ones (as for example the power network in a neighborhood). Furthermore, our development is extensible: it was obtained by extending the simple electrical network to a larger energy network, really a multiplex network [7], and it could be expanded further, to try to model even more complex systems, such as so-called sociotechnical systems [8].

\section{Power grid evolution}

The electricity grid has been in continuous evolution, from its beginning in the early nineteenth century until now and probably will do so in the future, affecting to its structure and elements.

In the classical grid, electric generators located at power stations obtain electric energy with high signal quality from classical energy sources. The transmission lines carry the electric power flow from generators to consumers, and the transformers serve to step-up for transmission and step-down for consumption of the energy [9]. The electric power flow is controlled in a centralized way and prices are fixed to be constant over long periods by the supply company.
This situation is changed and now, due to the use of renewable energy resources (solar, wind, biomass, etc.), the electrical distributed generation is characterised by high level of intermittency and poor signal quality, which strengthens the need to take action to compensate. To make better use of the discontinuous generation, two possibilities exist:

1. Establishing, demand-side management to seek plugging the more powerful loads in periods with higher generation and disconnect them during the lowest generation ones.

2. Storing excess energy produced during periods of peak generation and use when it is needed.

The first possibility has been the object of modeling in some of the authors previous works [10, 11, 12]. Clearly, a better performance would be obtained if the two possibilities are used simultaneously [13].

\subsection{Microgrids}

A microgrid is a cluster of electricity users and microsources that operate as a single controllable system for generating and using power. It encompasses a variety of distributed generation (DG), distributed storage (DS) and end-use loads. The microgrid enables the production and storage of renewable energy, as well as the exchange of electricity between energy providers and consumers, to take place locally. Microgrids can be considered as autonomous subsystems inside the powergrid. They can operate as "islands", but their most prominent common perspective is the integration of DG. Previous work of the authors on microgrids can be found in $[11,14,15,16,17,18]$.

\subsection{The smart grid}

The increasing incorporation of new elements of information and communication technologies (ICT), such smart meters, confers a much more complicated structure to the network, and must now be considered as a complex system of systems [17], the so called Smart-Grid [12, 19].

\subsection{The future energy network}

In descriptions of the Smart-Grid, only electrical elements are invariably considered. But, as indicated in Section 1, many directives and recommendations are aimed at improving the energy efficiency in production, transport, storage, transform and consumption processes. This makes the modeling process very attractive since experimenting on systems of systems is not generally feasible. It also makes modeling rather complicated because as described later, new elements to transform and store energy, and more networks in addition to the electrical one, for other energy carriers, like water, gas, fuel, etc., must be considered.

In this paper we build upon a published approach in order to develop a model for multi-carrier energy networks: the intelligent hub [18], used in electrical grid modeling, 
the aim has been to extend its functionality for multicarrier energy computational approaches such as Energy Hubs [6].

\section{Model conceptualization and design}

When trying to model the electricity grid one has two ways: first using specific electrical engineering tools and second using non specific mathematical tools, mainly from graph theory [20, 21], or computational, using object oriented programming, agent-based modeling, network modeling and simulation. In the first case the approaches have been successful for modeling the classical grid. But problems arise when trying to model the current grid because those tools are not enough to cope well with its real situation nor with the future Smart Grid. Some reasons are:

1. Possible switching behavior of generators and loads, due to renewable distributed generators and demand side management, respectively.

2. Event driven behaviors of generators and loads

3. Network structural changes. This is evident in operations of connection and disconnection of a microgrid, and also when accidental or catastrophic failures occurs.

4. Definition of a more powerful data structure for nodes. It becomes necessary in order to model some more powerful nodes [13] for the Smart Grid, with capacity to hold more data than the complex voltage value.

5. Working with interdisciplinary teams usually requires more general tools than the specialist ones used by electric engineers.

So some years ago we proceeded the second way. With practically no classical electrical software being reusable, our first attempt was to represent the electricity network using the mathematically correct elements and modeling them using object oriented, agent-based software [14], hoping so intending the models would be generalized to different situations and instances which could appear in the electrical network. For this, an important aspect, in our opinion, was for the grid model elements to be as simple as possible. Other researchers attempted simulation of the envisioned real grid, using aggregate models of actual components (even including electronic and communication components) [22], into multi-agent models. In the authors opinion those methods can be used as design approaches facing the implementation (for mobile robotics for instance) but are not a very convenient way for large simulation models, because that way the agents contains unnecessary details (like communication protocols for example) and the resulting model can became very large [23]. It seems interesting to note that some time ago, a growing number of authors were studying the Smart Grid in the context of complex systems [24, 18, 17], with two different visions in their treatments: a top-down, deterministic, based on the physical laws laws governing the system, and another less deterministic, bottom-up, suitable to agentbased models and able to produce the emerging dynamics during simulation. However, we think these approaches are not independent but are closely interrelated and in fact they constitute the essence of both real life and the model.

So the new method we propose here is a top-down design of the network structure and all and each one of its components, using simplified models, and a bottom-up aggregation of all of them into a multi-agent model describing the grid. We highlight the use of the bottom-up approaches to investigate future 'what-if' scenarios, allowing the user to modify and extend the load simulation using recognizable components.

\subsection{Electric circuit}

An electric circuit or electrical network is represented by a weighted graph $(V, E)$ where $V \subset \mathbb{N}$ is the set of vertices or nodes and $E \subset V \times V$ is the set of edges. Moreover, a weight, i.e. a scalar, real number (resistance) or complex number (impedance) is associated with each edge. Its electrical behavior is completely determined: if given voltages are applied at some (border) nodes, then currents circulate through the edges, and the other (interior) nodes acquire voltages, which can be calculated by Ohm and Kirchhoff laws, and also using probabilistic random graph methods $[25]$.



Figure 1: Electric network

A typical problem in electrical networks [21, p.59] is thus: given the voltage values at some network nodes, typically named sources or generators, then obtain the voltage values at all other nodes in the network. Let us illustrate it with an example. Figure 1 shows an electrical network with 14 nodes, where the resistance value of each connection across a couple of nodes is assumed to be equal to 1. The problem posed is, given the voltage values at the boundary nodes, we are asked for the voltage values at the internal nodes [25].

A right solution to the problem is obtained using standard electrical circuits like the so called mesh or node 
methods, based on the Ohm and Kirchhoff laws, that in essence consist of solving a system of linear equations. The solution for the voltage values, as can be seen in [25, p.22], is

$$
\left[\begin{array}{l}
v_{a} \\
v_{b} \\
v_{c} \\
v_{d} \\
v_{e}
\end{array}\right]=\left[\begin{array}{l}
0.8230 \\
0.7865 \\
0.8764 \\
0.5056 \\
0.3230
\end{array}\right]
$$

Another way to address the problem is related to the (original) Dirichlet problem, i.e., the problem of finding a function, solution of the Laplace partial differential equation

$$
\frac{\partial^{2} u}{\partial x^{2}}+\frac{\partial^{2} u}{\partial y^{2}}=0
$$

in the interior of a given region and that takes prescribed values on its boundary. The solutions of this problem are named harmonic functions.

An important property of harmonic functions is that the value $u(x, y)$ is equal to the average of the values over any circle with center $(x, y)$ lying inside the region [25, p.17]. Physically, this property is closely related to the principles of mass/matter conservation and continuity of flow.

For the discrete Dirichlet problem the function $u(\cdot)$ takes values at the nodes of a network (weighted graph), and if all the weights are equal to one the property can be expressed as: the value $u_{v}$ at any internal node $v$ equals to the average value at all nodes connected to it:

$$
u_{v}=\frac{\sum_{x \in C_{v}} v_{x}}{\left|C_{v}\right|}
$$

where $C_{v}$ is the set of the nodes connected to the node $v$. In general, if the weights are scalar, real or complex numbers, it is easy to show that

$$
u_{v}=\frac{\sum_{x \in C_{v}} \frac{v_{x}}{Z_{x}}}{\sum_{x \in C_{v}} \frac{1}{Z_{x}}}
$$

where $Z_{x}$ is the weight of the edge $(v, x)$, called impedance in case of electrical networks.

The relaxation method consist of starting from an initial value at any internal node and applying iteratively the rule, to calculate the value at each internal node. Note that the iteration is carried out only on the interior nodes.

It is known that the sequence of values at each node converges to the solution of the Laplace equation.

In the case of the electrical network circuit shown above (Figure 1) the values calculated by the relaxation method converges to the previously calculated ones. For this, we start the iteration from node $a$, following to $b, c, d, e$.
Then, for the first iteration, $i=1$, we have

$$
\begin{aligned}
& v_{a}=\left(v_{b}+1+1+v_{d}\right) / 4=(0+1+1+0) / 4=0.5 \\
& v_{b}=\left(1+1+v_{a}+v_{e}\right) / 4=(1+1+0.5+0) / 4=0.625 \\
& v_{c}=\left(v_{d}+1+1+1\right) / 4=(0+1+1+0) / 4=0.75 \\
& v_{d}=\left(v_{e}+v_{a}+v_{c}+0\right) / 4=(0+0.5+0.75+0) / 4=0.3125 \\
& v_{e}=\left(0+v_{b}+v_{d}+0\right) / 4=(0+0.625+0.3125+0) / 4=9375
\end{aligned}
$$

After iteration $i$ finishes, the next iteration is computed using the same formulae but using the variable values calculated in the previous one. Table 1 shows the results from 10 iterations.

\begin{tabular}{r|ccccc}
$i$ & $v_{a}$ & $v_{b}$ & $v_{c}$ & $v_{d}$ & $v_{e}$ \\
\hline 1 & 0.5000 & 0.6250 & 0.7500 & 0.3125 & 0.2344 \\
2 & 0.7344 & 0.7422 & 0.8281 & 0.4492 & 0.2979 \\
3 & 0.7979 & 0.7739 & 0.8623 & 0.4895 & 0.3159 \\
4 & 0.8159 & 0.7829 & 0.8724 & 0.5010 & 0.3210 \\
5 & 0.8210 & 0.7855 & 0.8753 & 0.5043 & 0.3225 \\
6 & 0.8225 & 0.7862 & 0.8761 & 0.5052 & 0.3229 \\
7 & 0.8229 & 0.7864 & 0.8763 & 0.5055 & 0.3230 \\
8 & 0.8230 & 0.7865 & 0.8764 & 0.5056 & 0.3230 \\
9 & 0.8230 & 0.7865 & 0.8764 & 0.5056 & 0.3230 \\
10 & 0.8230 & 0.7865 & 0.8764 & 0.5056 & 0.3230
\end{tabular}

Table 1: Node voltage values at each iteration

As it can be seen, after the 7-th iteration the values converge to those calculated before (with 4 decimal digits of precision).

The relaxation method is decentralized, since the calculus process is made locally at each node, using only the data of nodes connected to it, so it is especially well suited to be used in agent based models, as we will see in section 5 .

\subsection{Flow networks}

As later in this article we will need to model not only electricity networks but also other fluid distribution networks, such as gas, water, etc., used in facilities in cities and industry, in this section we will try to review some important schemes and methods useful for their calculation and modeling.

An electrical network, or circuit, is an special case of a more general kind of networks, the so called flow networks, also known as transport networks. Such networks have been long studied by Graph Theory [20], being one of its first and most important applications. A flow network can be used to model a number of real systems such as electric circuits, road traffic, fluids through pipes, etc.

In this theory, a flow network is usually represented by a weighted graph $G=(V, E)$, where $V=\{1, \ldots n\}$, $E \subset V \times V$, in which a real (or complex) number $v_{i}$ is assigned to each vertex $i$ and another real (or complex) number $e_{i j}$ is assigned to each edge $(i, j)$, for $1 \leq i, j \leq n$. Some times vertices and edges are called nodes and arcs, 
respectively, and also other special names in some applications. The variables associated with the vertices and edges are often called across and through variables, respectively. Every vertex $(i)$ in $G$ is associated with a number $e_{i}$ (across variable) representing the effort at the corresponding vertex, and every edge $(i, j)$ is associated with a number $f_{i j}$ (through variable) representing the flow passing through the edge. It is known that the product of these two variables is power [26]:

$$
p_{i j}=f_{i j}\left(e_{i}-e_{j}\right)
$$

Figure 2 shows a flow network $G$ with $n+1$ vertices (a further node $e_{0}$ has been used) in which some vertices and edges are drawn. Usually arbitrary directions are assigned to flows and then the graph becomes a digraph.

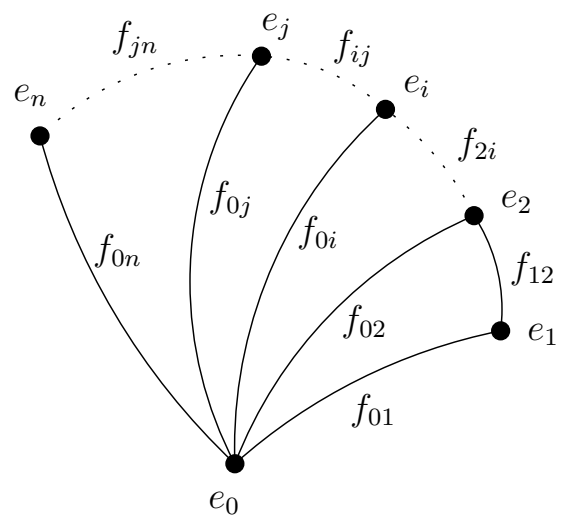

Figure 2: Network graph

In the different technological areas, constitutive relations give the relations between the across and flow variables, such as $f_{i j}=\varphi\left(e_{i}, e_{j}\right)$, that some times can be considered as linear $f_{i j}=c_{i j}\left(e_{i}-e_{j}\right)$ or quadratic $f_{i j}=$ $d_{i j}\left(e_{i}^{2}-e_{j}^{2}\right)$. If we assume linear constitutive relations, we have

$f_{0}=\left(e_{0}-e_{1}\right) c_{01}+\ldots+\left(e_{0}-e_{i}\right) c_{0 i}+\ldots+\left(e_{0}-e_{n}\right) c_{0 n}$

$f_{1}=\left(e_{1}-e_{0}\right) c_{10}+\ldots+\left(e_{1}-e_{i}\right) c_{1 i}+\ldots+\left(e_{1}-e_{n}\right) c_{1 n}$

$f_{i}=\left(e_{i}-e_{0}\right) c_{i 0}+\ldots+\left(e_{i}-e_{j}\right) c_{i j}+\ldots+\left(e_{1}-e_{n}\right) c_{i n}$

$f_{n}=\left(e_{n}-e_{0}\right) c_{n 0}+\ldots+\left(e_{n}-e_{i}\right) c_{n i}+\ldots+\left(e_{n}-e_{n-1}\right) c_{n, n}$

where

$$
f_{i}=\sum_{\substack{j=0 \\ j \neq i}}^{n} f_{i j}
$$

The left hand side is the given net flow injection at each node, and the equation is based on the law of conservation of flow. The system of equations can be written in matrix notation as

$$
\left[\begin{array}{c}
f_{0} \\
f_{1} \\
\vdots \\
f_{i} \\
\vdots \\
f_{n}
\end{array}\right]=\left[\begin{array}{cccccc}
c_{00} & -c_{01} & \ldots & -c_{0 i} & \ldots & -c_{0 n} \\
-c_{10} & c_{11} & \ldots & -c_{1 i} & \ldots & -c_{1 n} \\
\vdots & \vdots & \ddots & \vdots & & \vdots \\
-c_{i 0} & -c_{i 2} & \ldots & c_{i i} & \ldots & -c_{i n} \\
\vdots & \vdots & & \vdots & \ddots & \vdots \\
-c_{n 0} & -c_{n 2} & \ldots & -c_{n i} & \ldots & c_{n n}
\end{array}\right]\left[\begin{array}{c}
e_{0} \\
e_{1} \\
\vdots \\
e_{i} \\
\vdots \\
e_{n}
\end{array}\right]
$$

where

$$
c_{i i}=\sum_{\substack{j=0 \\ j \neq i}}^{n} c_{i j}
$$

The matrix $L=\left[c_{i j}\right]_{0}^{n}$ is named the Laplacian Matrix, or Kirchhoff Matrix, of the graph $G$. If as usual, one of the nodes, for example node 0 , is used as reference node, we have

$$
e_{0}=0
$$

and then

$$
\left[\begin{array}{c}
f_{1} \\
\vdots \\
f_{i} \\
\vdots \\
f_{n}
\end{array}\right]=\left[\begin{array}{ccccc}
c_{11} & \ldots & -c_{1 i} & \ldots & -c_{1 n} \\
\vdots & \ddots & \vdots & & \vdots \\
-c_{i 2} & \ldots & c_{i i} & \ldots & -c_{i n} \\
\vdots & & \vdots & \ddots & \vdots \\
-c_{n 2} & \ldots & -c_{n i} & \ldots & c_{n n}
\end{array}\right]\left[\begin{array}{c}
e_{1} \\
\vdots \\
e_{i} \\
\vdots \\
e_{n}
\end{array}\right]
$$

that is

$$
F=C E
$$

and $f_{0}=\sum_{i=1}^{n} c_{0 i} e_{i}$. It is easy to see that for quadratic constitutive relations one gets

$$
F=D \tilde{E}
$$

where $D=\left[d_{i j}\right]_{0}^{n}$ and $\tilde{E}=\left[e_{j}^{2}\right]_{0}^{n}$.

Equations (4), (5) or other obtained for different constitutive relations, can be used for analysis and calculus in different areas. Note that in order to obtain the diagonal elements $c_{i i}$ of matrix $C$, the elements $c_{i 1}, i=1, \ldots, n$ in the first column of matrix $L$ are also needed.

\subsection{Modeling the electrical grid}

Engineers use grid models to estimate the voltages and currents anywhere in the circuit. If voltages at all the network generators (or current sources) are given, then a linear system results and mesh (or nodal) analysis can be used to solve the problem using standard linear algebra methods, or even the relaxation method described in Section 3.1 .

But in the most usual problem in electrical power systems engineering, the statement is different and it leads to a non-linear system of equations. In this case, the most common method used is Power Flow Analysis. This method is explained in detail in classical power system books [27, 28] and indeed we could use the formulas that 
appear in them. But instead we are interested in deducing the appropriate expressions from (4), since we aim to solve the problem using an agent based model and also to obtain an homogeneous formulation for all types of energy carriers.

\subsection{Power Flow}

As it is well know, in alternative current analysis (AC), using phasorial notation, the apparent power results to be

$$
\mathbf{s}=\mathbf{v} \mathbf{i}^{*}
$$

Expressions (3) and (4), valid for networks carrying any type of energy carrier, for electrical networks can be written as

$$
\left[\begin{array}{c}
\mathbf{i}_{1} \\
\vdots \\
\mathbf{i}_{i} \\
\vdots \\
\mathbf{i}_{n}
\end{array}\right]=\left[\begin{array}{ccccc}
\mathbf{y}_{11} & \ldots & -\mathbf{y}_{1 i} & \ldots & -\mathbf{y}_{1 n} \\
\vdots & \ddots & \vdots & & \vdots \\
-\mathbf{y}_{i 2} & \ldots & \mathbf{y}_{i i} & \ldots & -\mathbf{y}_{i n} \\
\vdots & & \vdots & \ddots & \vdots \\
-\mathbf{y}_{n 2} & \ldots & -\mathbf{y}_{n i} & \ldots & \mathbf{y}_{n n}
\end{array}\right]\left[\begin{array}{c}
\mathbf{v}_{1} \\
\vdots \\
\mathbf{v}_{i} \\
\vdots \\
\mathbf{v}_{n}
\end{array}\right]
$$

that is the Ohm law,

$$
\mathbf{I}=\mathbf{Y V}
$$

where $\mathbf{V}, \mathbf{I} \in \mathbb{C}^{n}$ are the voltages and intensities in the network (across and through variables) and $\mathbf{Y} \in \mathbb{C}^{n \times n}$ is called the admittance matrix.

If $\mathbf{V}$ is given (and also if $\mathbf{I}$ is given), equation 8 represents a linear system of equations and then the solution can be obtained using standard linear algebra methods or relaxation methods. But it is not the case because for typical electrical network problems, three different kind of buses exist, each with different specified variables, and then a non-linear system of $2 n$ equations on $2 n$ real unknowns results. Let us briefly consider the matter.

\subsubsection{Power Flow calculus}

Each bus in a power system can be classified in one of three types Slack, Control and Load, each denoted by symbol, as Figure 3 show, for the schematic representation commonly used in wiring diagrams, books and papers on electrical engineering. Sometimes they are also called, Reference or Swing, PV and PQ, respectively. The difference between these buses is due to the known and unknown magnitudes assigned to them are different:

0. Slack bus - voltage magnitude and phase are known active and reactive powers are unknown

1. Control bus - voltage magnitude and active power are known - phase angle and reactive power are unknown

2. Load bus - active and reactive power are known - voltage magnitude and phase are unknown

where a bus index $0,1,2$, is used to refer to them.

Some hypotheses assumed are that only one slack bus exists in the network, and any number of control and load



Figure 3: Bus types

buses can be attached at each bus, giving a mixed bus in this case, as Figure 3 shows, and then the net power, i.e. the arithmetic sum of power of all connected buses to this bus, must be calculated at initialization. Note this mixed bus is able to model a number of actual node states, like any mix of generators and loads, very useful in renewable distributed generation, and also energy storage handling, by letting negative values to the associate flow for store energy and positive ones for use it, acting then as generators.

The slack bus acts automatically as source/sink, i.e. it is able to generate or sink all the amount of power generated or consumed at all other buses in the network.

Usually a per-unit representation is used. That means that each actual magnitude $X$ at some element in the system is expressed as a fraction

$$
X_{p u}=\frac{X}{X_{\text {base }}}
$$

of some base magnitude $X_{\text {base }}$ fixed for each element (usually its nominal value). Using this notation, calculus becomes easier and schematics are simplified because some elements (like transformers) disappear from the circuit, which then can be represented as a standard graph.

An iterative process follows. For each $i$-th bus, if bus type is 2 , given $p_{i}$ and $q_{i}$ then the complex number $\mathbf{v}_{i}$ must be calculated. From Equation (7), the intensity injection at bus $i(i=1, \ldots, n)$ is

$$
\mathbf{i}_{i}=\sum_{k=1}^{n} \mathbf{y}_{i k} \mathbf{v}_{k}=\mathbf{v}_{i} \mathbf{y}_{i i}+\sum_{\substack{k=1 \\ k \neq i}}^{n} \mathbf{y}_{i k} \mathbf{v}_{k}
$$

and since Equation (6) yields

$$
s_{i}^{*}=\mathbf{v}_{i} \mathbf{i}_{i}^{*}=p_{i}-j q_{i} \Rightarrow \mathbf{v}_{i}^{*} \mathbf{i}_{i}=p_{i}-j q_{i} \Rightarrow \mathbf{i}_{i}=\frac{p_{i}-j q_{i}}{\mathbf{v}_{i}^{*}},
$$

substituing $\mathbf{i}_{i}$ into Equation (9), the complex voltage value at bus $i$ equals

$$
\mathbf{v}_{i}=\frac{1}{\mathbf{y}_{i i}}\left(\frac{p_{i}-j q_{i}}{\mathbf{v}_{i}^{*}}-\sum_{\substack{k=1 \\ k \neq i}}^{n} \mathbf{y}_{i k} \mathbf{v}_{k}\right)
$$

If bus type is 1, Equation (11) is not directly applicable because the reactive power $q_{i}$ is unknown. But, since voltage $\mathbf{v}_{i}$ is given, then from Equations (9) and (10) an estimate of it can be performed, 


$$
q_{i}=-\operatorname{Im}\left(v_{i}^{*} \sum_{k=1}^{n} \mathbf{v}_{i k} \mathbf{v}_{k}\right),
$$

and after that, Equation (11) can also be used.

And finally, if bus type is 0 , Slack bus (usually with $i=1$ ), the active and reactive powers, $p_{i}$ and $q_{i}$, can be calculated, using (9) and (10). The above rules are usually implemented in the so called Gauss-Seidel algorithm [27].

This process is computed by the agents in the model, in a decentralized manner. Each agent computes its "rule", i.e. the complex voltage value $\mathbf{v}_{i}$ at each $i$-th node is computed and then it is immediately used as actual value for calculating the new voltage value $\mathbf{v}_{i+1}$ at the next agent. In this way, from the aggregate of all agents emerges the behavior of the network, that is, the flow of power through the lines. It is interesting to note that sequence order of agents is not determinant, any can be valid, and for this reason it is well suited to cope with possible sudden changes in the network.

\section{Modeling energy networks}

Today the most extended and used energy network is the electric one where the electrical energy is transmitted from node to node through electrical lines. As it was showed before, an electrical network can be mathematically represented by a weighted graph $G=(V, E)$, where $V$ is the set of vertices and $E$ is the set of edges in the network andthe power flow algorithm is usually used to calculate the electrical voltages at the nodes and currents or powers flowing through the edges.

A multi-carrier energy network consist of a number of energy conductors, through which different kind of energy flows between the network nodes, usually using special fluids called energy carriers, where they can interact with each other and the environment. The idea to model these kind of networks is to exploit as much as possible the elements of electrical networks, trying to use them for other energy carriers and then combine them in a multi-carrier network. For doing so, a re-thinking of the node and edge concepts, using the $H u b$ concept developed in [6], with a special version of the multi-agent power-flow algorithm developed by the authors $[18,17]$ to extend it to the multicarrier case, has been implemented.

Some part of the work has been done already and showed before because the main the previously calculated formulae are valid not only for electrical systems, but also for any other kind of energy carriers.

\subsection{Energy kinds}

Electrical power is not the only energy carrier. Gas and heat utilities are very useful to cover domestic energy demand. In the gas utility, gas flows through pipes from some generator nodes where the fluid is pushed into the network to other consumer nodes where the chemical energy in the gas is transformed into heat for use it. In

\begin{tabular}{l|c|c|c} 
Energy kind & energy carrier & energy conductor & color \\
\hline Mechanical (m) & none & shafts and pulleys & black \\
Electrical (e) & electricity & wires & red \\
Chemical (g) & gas or liquid & pipes & green \\
Thermal (h) & gas or liquid & pipes & blue \\
Communications & digital signal & wires-air-vacuum & gold \\
\hline
\end{tabular}

Table 2: Energy kind

the heat utility, usually water (sometimes as steam) flows through pipes from generators to consumers, where the thermodynamical energy in the gas is transformed to heat and used.

Another way to deliver energy that can be seen in books are old systems of mechanical power transmission, widely used during the Industrial Revolution, which, although not widely used today as heating services, are often used as subsystems within other facilities. These single mechanic systems do not use fluids nor pipes but energy goes directly from generator nodes to consumer nodes through a system of shafts and pulleys.

These are probably the four kinds of energy most used in existing facilities. There are, of course, other kind of possible energy transmission, such as electromagnetic energy through air or special media, light energy (sun, laser), etc., but in this paper, we will use only those four.

Moreover, although it is not really an energy transmission in the usual sense, a further kind of energy, communication, has been added. It represents digital signals flowing through some media to send messages - usually related to events - between the network nodes, and it is responsible for the event driven digital changes in the network.

In this way, five energy kinds will be considered, as Table 2 summarises. This gives the network a layered structure, with each energy kind flowing in a different layer, which is not far from reality.

In order to obtain a rather general modeling approach, no special technological data is given to define each object. That is possible because, by the multi-agent based method used, agents are defined as Java classes with only some general and structural properties defined, being the special ones left to the last part, at model instantiating time. In this way the models may be used to cover a wide sector of infrastructure utilities, including not only the traditional ones (electricity, natural, gas and heat) but others, having the same father classes, can be created as different instances of it. So for example, the generic Chemical Energy class can be used to model any kind of gas, not only natural gas and fossil gas but even hydrogen energy networks. Also the Heat class, which could serve to model a number of utilities such as heating (water as carrier), air conditioning (cold air as carrier), geothermic energy, pump heath, industrial facilities that use hot oil, etc.

The method followed for modeling was to mimic the previously described electrical power-flow method for the other energy carriers and change the value of potential at each node according to its type. But the problem is 
simplest in cases where the carrier is not electricity because then the known and unknown variables are real and besides there are only Slack type generators.

The symbols used in diagrams can be also borrowed from the electric ones if some special notation is used for denoting them. Figure 4 shows a possible notation for Slack and Load buses for mechanical, chemical and thermal energies, where the Control and Load labels have been obviated, because their icons denote them.
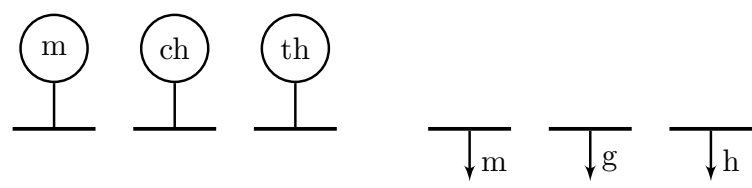

Figure 4: Energy bus types

Then the power flow algorithm is still valid, but can be simplified enough. It is easy to see that in these cases, it is equivalent to the relaxation method explained above.

Note that the power-flow algorithm allows us to consider the multi-carrier energy network as a number of $i n$ dependent graphs, at different layers, because the values only change at the times when the algorithm steps. For a continuous time analysis, a further assumption, perhaps as weighted and edge coloured graphs should be necessary and also the "small" networks into each node should be modeled.

In this way the model results as the superposition of a number of networks, one for each energy carrier, which one can imagine as if were "glued" at some nodes. Actually, if we refine the model we will see each node like a network, where the electric carriers interact. So in this way the model can be seen as a network of networks where the "big" network is the main network and each node is a "small" network, functioning as a conversion point [29], as it is explained below.

The power-flow algorithm is valid to calculate the electrical magnitudes and also, once adapted, serves also to the other fluids, in fact is easier because it does not requires complex calculus.

Figure 5 shows two graphs, the first one is a standard graph corresponding to a single energy network, and the second one is a graphical representation of a multi-carrier energy network.

\subsection{Dynamic network structure}

The classical electrical power flow assumes each network node is of a prefixed type, that is, generator type or load type. But in smart grids a number of generators and loads can be attached to each node and there exists the possibility that any node can change dynamically from some type to other, in a continuous way or driven by events, so this should be taken into account for modeling. The same situation is true, for each energy carrier, for energy networks. So each hub, having a number of carriers, may have exhibit


Figure 5: Single and multi energy carrier network graphs

\begin{tabular}{|c|c|c|c|c|c|c|c|c|}
\hline $\begin{array}{c}\text { Energy } \\
\text { carrier }\end{array}$ & \multicolumn{7}{|c|}{ Hub types } \\
\hline elec & G & G & G & G & L & L & L & L \\
gas & G & G & L & L & G & G & L & L \\
heat & G & L & G & L & G & L & G & L \\
\hline
\end{tabular}

Table 3: Different possibilities of bus types

a number of possible combinations. For instance, for three energy carriers, electricity, gas and heat, and two type, G (generator) and L (load), of node behavior for each, the number of possibilities is eight, as can be seen in Table 3 . Eight $\left(2^{3}\right)$ types of node need to be accommodated in a model with three energy kinds, and $16\left(2^{4}\right)$ for four, and so on. This would require to model eight different kind of node types, or more if the number of carriers is bigger.

\subsubsection{Energy Hubs}

The conceptual approach of hybrid energy hub [30] acts as an interface between energy producers, consumers, and the transportation infrastructure. It is a unit that provides the basic features of input and output, conversion, and storage for multiple energy carriers, acting as an interface between the network agents (producers, consumers, infrastructures), by coupling their connection lines. So the energy hub represents a generalization or extension of the network node in an electrical system [6].

A hub can be imagined as a block provided with input and output terminals for energy conductors, and also internal energy storage elements. Due to the high number of real energy carriers (electricity, gas, water, hydrogen, etc.), and also to their different possible combinations, a huge variety of energy hubs can be imagined and in fact many 
are used in industrial and domestic applications. Therefore, with the aim of simplifying the analysis, which uses output from the modeling, we were parsimonious with the number of energy carriers based on the notion of modeling energy kinds corresponding to each of the carriers instead of using energy carriers themselves. This implies increasing the level of abstraction of the model, leaving the specific choice of the energy carriers for the final stages of implementation, where data and real characteristics of all elements should be assigned.

\subsection{Energy conversion}

Energy of some kind can be converted to another kind through a special conversion process, with some losses rate given by a efficiency factor $\eta$. This often occurs in nature, for instance in the efficient photosynthesis process, through which plants absorb energy from light (sun energy) to reduce $\mathrm{CO}_{2}$ into sugars (chemical energy), like in Figure 6. Also a number of different kind generators can be combined, as Figure 7 shows, creating different energy kinds from different energy sources.

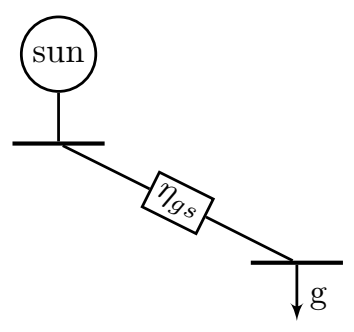

Figure 6: Energy conversion

One can imagine that five sockets are arranged into each hub to be used by the internal agents for communication, mechanical energy (m), electrical energy (e), chemical energy (g) and thermal energy (h), respectively. From an energy point of view only the four last are operative, so the energy conversion process at each hub can be represented as a block with four inputs

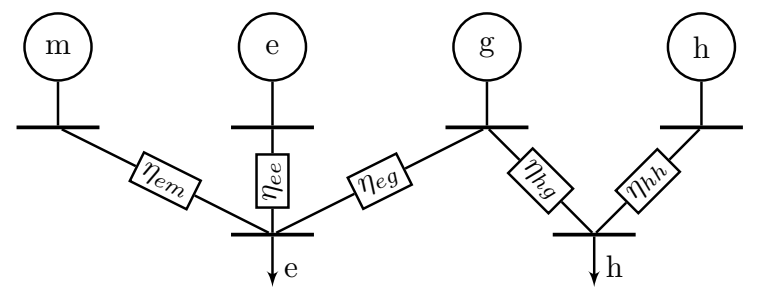

Figure 7: Energy conversion

$p_{m}, p_{e}, p_{g}, p_{h}$ for input power flows, and four outputs $l_{m}, l_{e}, l_{g}$ and $l_{h}$ for output load flows, as Figure 8 shows.
The relationships between them are given by

$$
\left[\begin{array}{c}
l_{m} \\
l_{e} \\
l_{g} \\
l_{h}
\end{array}\right]=\left[\begin{array}{cccc}
\eta_{m m} & \eta_{m e} & \eta_{m g} & \eta_{m h} \\
\eta_{e m} & \eta_{e e} & \eta_{e g} & \eta_{e h} \\
\eta_{g m} & \eta_{g e} & \eta_{g g} & \eta_{g h} \\
\eta_{h m} & \eta_{h e} & \eta_{h g} & \eta_{h h}
\end{array}\right]\left[\begin{array}{c}
p_{m} \\
p_{e} \\
p_{g} \\
p_{h}
\end{array}\right]
$$

or in matrix notation

$$
L=E P
$$

where $P$ is the input power flow vector, $L$ is the output load flow vector and $E$ is the hub efficiency matrix.

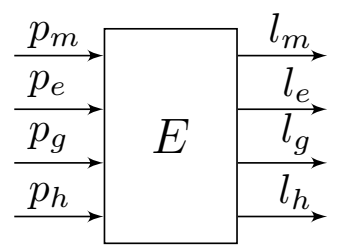

Figure 8: Energy conversions in a hub

\subsubsection{Storing energy}

Each energy carrier introduces energy of a given type in the hub, where it can be transformed into energy of another type, consumed or stored. Suppose for example that a flow $p_{e}$ of electrical energy comes through the wires into the hub, where a fraction of it is converted to another type of energy (flow $p_{e m}$ to mechanical energy, flow $p_{e g}$ to chemical energy, and flow $p_{e h}$ to thermal energy), another fraction $p_{e s}$ is stored and the remainder fraction $p_{e e}$ is consumed. Also it is possible that flows $l_{m e}, l_{g e}, l_{h e}$, coming respectively from mechanical, chemical and thermal conversions in other hubs, are feed, and also that a portion of electrical energy $l_{e s}$ is being stored after conversion, as Figure 9 shows.

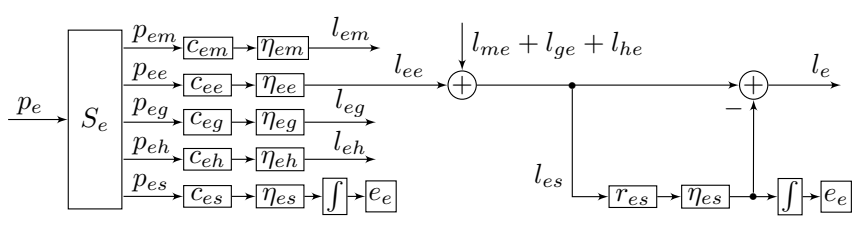

Figure 9: Electrical socket in a hub

Doing the same for the other energy kinds considered and joining them results the diagram shown in Figure 10.

A simpler and intuitive representation can be achieved using a graph like picture shown in Figure 12, where the dark zones at each node represent energy storage elements, one near the power input and the other near the load, the clear zones represent loads, and the coloured lines represent the conversion factors and efficiencies. One can realise that this diagram is close to a System Dynamics representation of the hub. 


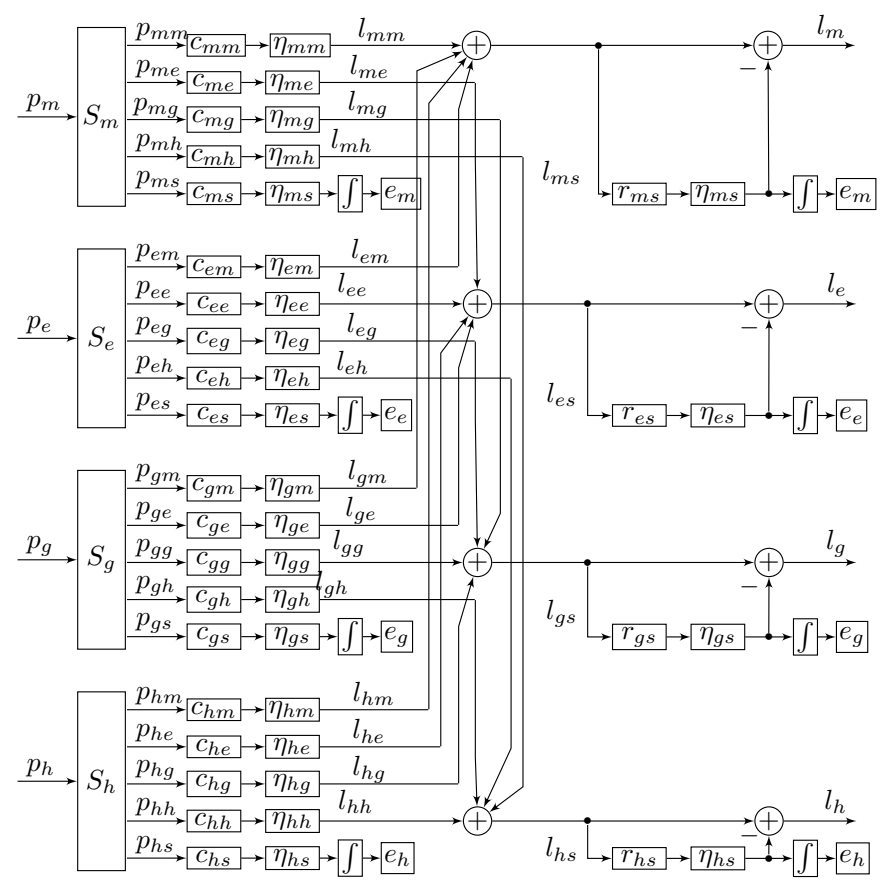

Figure 10: Hub internal diagram

As Figure 12 shows, there is a network inside each hub, so really each hub is a network, and therefore a multicarrier energy network can be considered as a network of networks. Even more, if we were to look very closely, we would see that each of the small pentagons at the vertices of this network is actually a new hub, and so on, so definitely we have a recursive structure of network of networks.



Figure 11: multi-carrier energy network graph

Figure 11 shows a possible multi-carrier energy network with six nodes where each of them is a multi-carrier energy hub, so each of the small pentagons corresponds to a hub as that of Figure 12.

\section{Model implementation}

The conceptualization and design for a multi-carrier energy network is implemented into an AnyLogic model as described below.

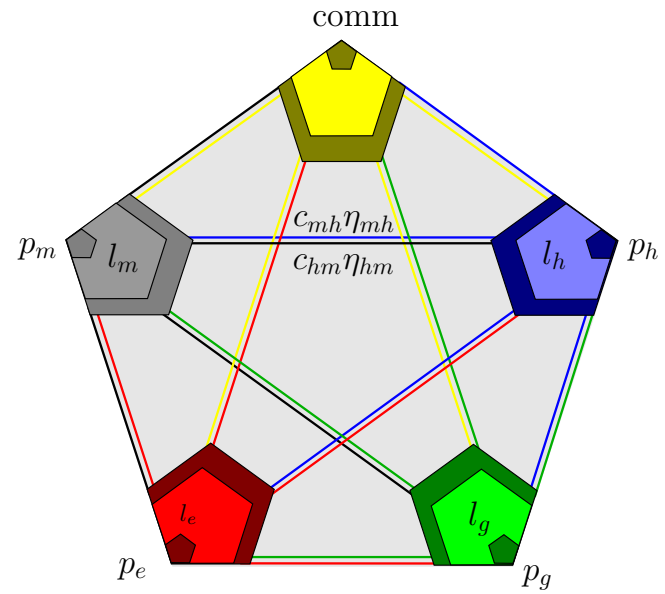

Figure 12: multi-carrier energy hub

\subsection{Agent based model of an electric circuit}

The implementation of the electric circuit of section 3.1 is described here. The model contains 14 agents representing the network nodes. At each simulation step, each agent of interior nodes $a, b, c, d, e$, computes $v$ as the average value of all of the nodes connected to it, implementing the relaxation method.

The model simulation window appears in Fig 13 showing the circuit and results.

This simple example is of particular interest, because the same or similar algorithm works not only for electric networks but also for other kinds of networks in which mass conservation or fluid continuity properties can be assumed.

\subsubsection{Agent-based power flow method}

The implementation of the Gauss-Seidel algorithm in AnyLogic is straightforward using the Gauss Seidel algorithm, based on Equations (9), (10), (11) and (12). The appropriate Java code is written for the agent active object class. To facilitate complex number calculations, we used the public domain library complex.java [31]. At each simulation step time, the model obtains the values of the active power and voltage at each generator and also the values of the active and reactive power at each load and then calculates the voltage at the load buses. In standard Power Flow calculations, the iteration stops when the absolute value of the difference between the last and the previous calculated voltage values is smaller than some prefixed tolerance $\epsilon$. But in the agent based model the iteration never stops while simulation is running, since the algorithm acts over agents at each simulation steps, when each wakes up.

For testing the model, the well known 6 -bus power system [32, p.104], with the same data, was used.

The values of voltages and powers at each bus, after the 21-st iteration can be seen in Figure 14. The model was also successfully checked against other standard test 


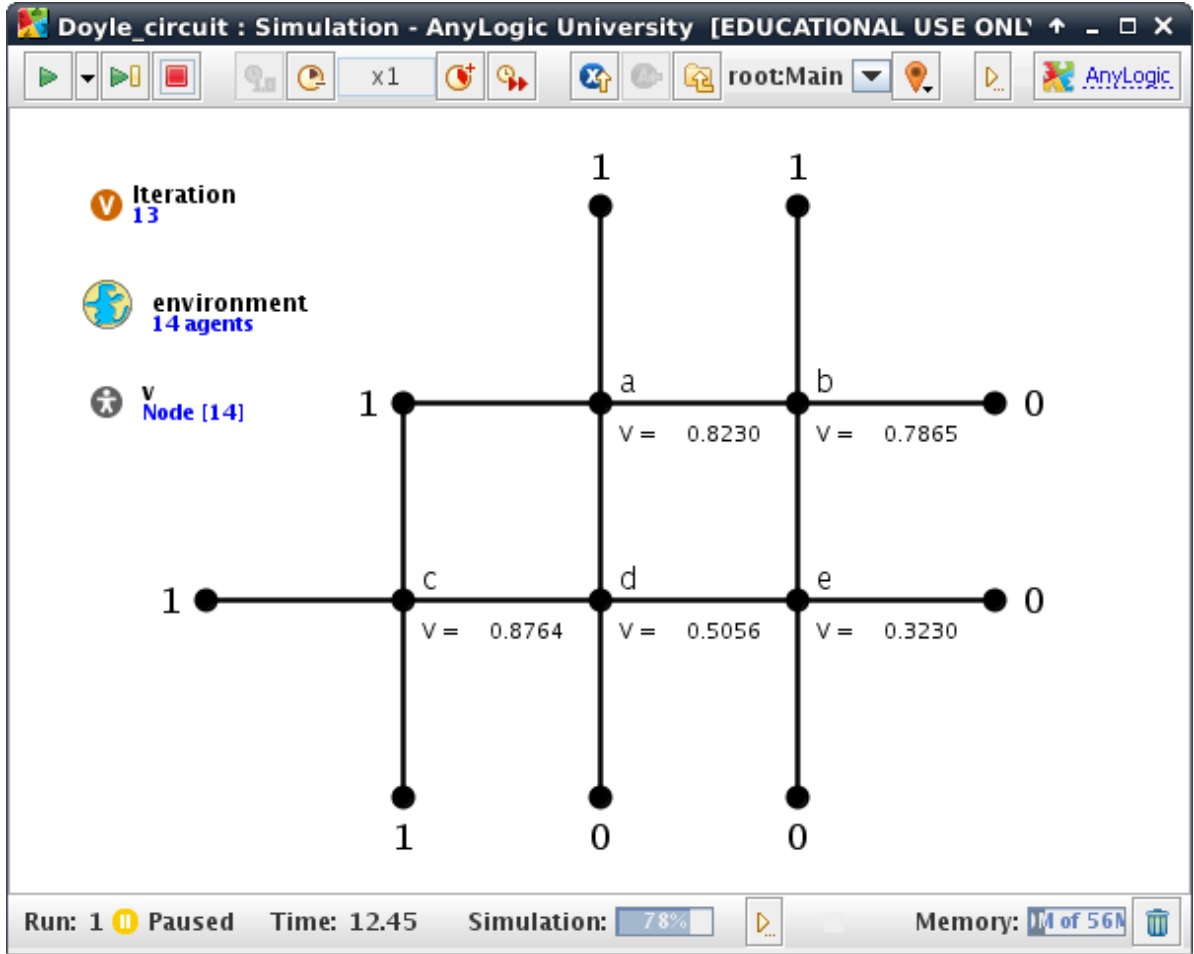

Figure 13: Electric network agent-based simulation

systems, like the IEEE 14 Bus System and the IEEE 30 Bus System.

In our first microgrid models $[14,15]$, the mesh method was used (see Section 3.1 of this paper) but in later papers $[17,18]$ the power flow method was incorporated. For this current paper we used the Gauss-Seidel method [33] due to its easy way to translate the standard algorithm to a decentralized form for agent-based modeling. Some modelers argue that convergence is very slow and most use the Newton-Raphson method [28], even for distributed control strategies modeling. However for agent-based modeling, each agent is continuously interacting with the other agents so the power flow iteration is only one among all the operations it has to perform at each iteration step, and therefore the speed of convergence is not so important. Researchers, from the United States Department of Energy, recently also used a Gauss-Seidel approach to develop the so called GridLAB-D [34], a rather powerful simulator for the US electrical distribution grid.

The implementation of the agent-based algorithm in Anylogic was successfully validated against a load flow calculation performed using the Matlab PSAT toolbox [35].

\subsection{Agent based model of an energy network}

We consider a number $n$ energy carrying fluids and $n$ energy networks, one for each fluid. We imagine the networks are glued at their nodes, shaping hubs composed by $n$ single nodes.

\begin{tabular}{l|cccccc} 
& \multicolumn{6}{|c}{ hindex $=$ colon } \\
\hline Hub & 0 & 1 & 2 & 3 & 4 & 5 \\
\hline Elec & 0 & 1 & 2 & 3 & 4 & 5 \\
Gas & & & 0 & 1 & 2 & 3 \\
Heath & & & & 0 & 1 & 2
\end{tabular}

Table 4: hindex variable

An object called Hub is created and $n$ agents of class Hub are created in the Main class, representing the network hubs. So the hub agents became the starred actors, acting at the network nodes, being responsible of changing the numerical values associated with the nodes as functions of the other values at the glued nodes.

A mechanism is needed to associate in the model the different carrier agents which are glued to each hub agent. An obvious way could be to introduce the carrier agents into each hub agent, but this resulted in difficulties when applying the power-flow algorithm. An alternative index method was implemented, using a Java variable hindex, into each agent. For simplicity we implemented a threecarrier energy network, for the electricity, gas and heat, as shown in Figure 15. Each hub has been obtained by gluing a maximum of three different agents. But for the powerflow algorithm to work properly, the relative position of each agent must be indicated.

Table 4, which follows from Figures 15 and 16, gives the relative positions of agents: the number at each cell in 


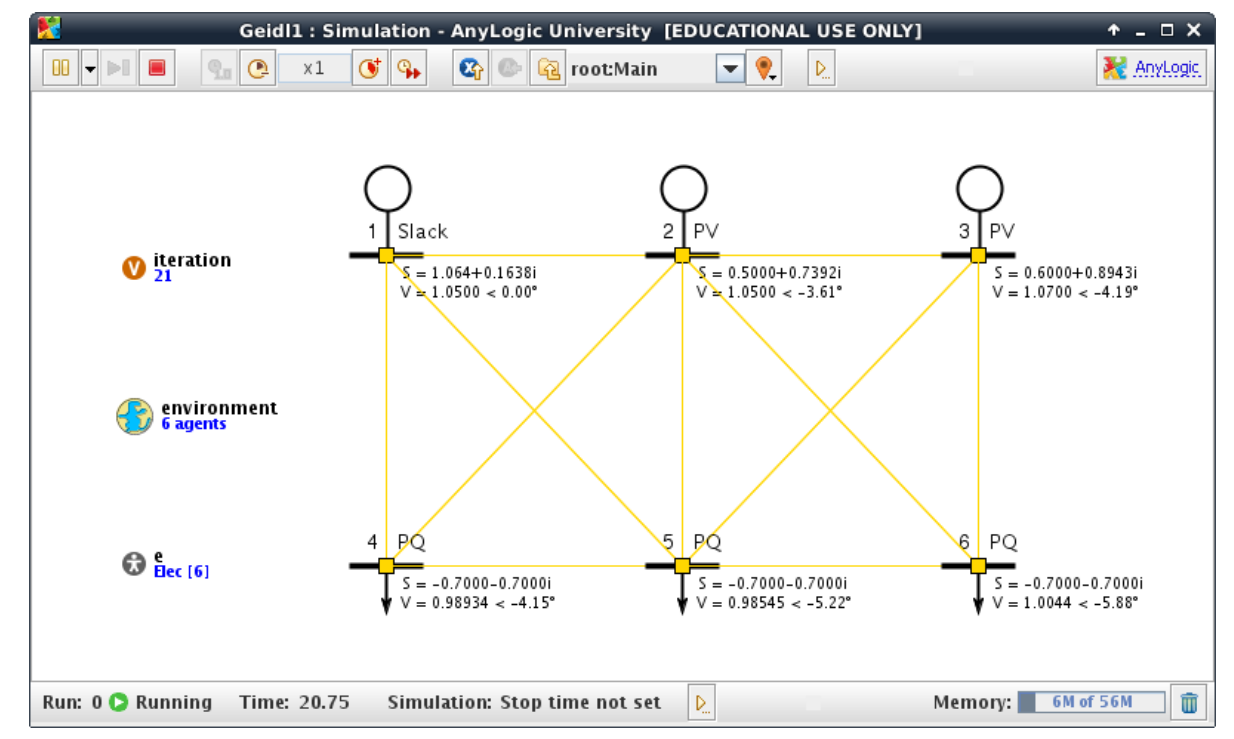

Figure 14: Agent-based power flow simulation

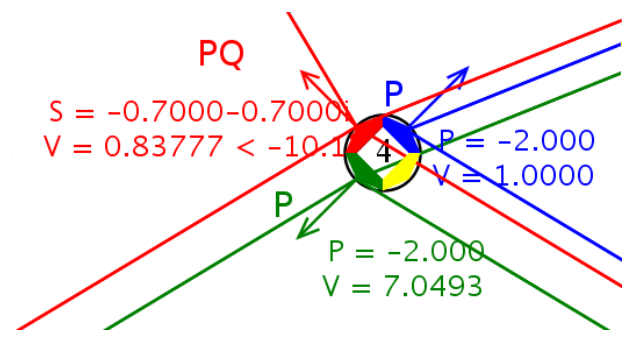

Figure 15: Energy Hub instance in AnyLogic simulation

that table represent the respective agent number, and its colon number indicates the index of the hub to which it is glued. Note there is a offset by 1 at the hubs numbers represented in Figure 16, in respect of indices in Table 4 that shows their associate Java integer variables, starting at zero.

One can imagine these glued hubs like sockets in the model where perhaps some other agents representing people can plug and unplug appliances and other energy consumer elements.

\section{Scenario}

With the above we have created a model for the structure and power flow in a multi-carrier energy network, which can be used to calculate potentials at nodes and flows through edges of the network, in terms of power generating devices and loads or energy consumers attached to each node.

Then, in order to model any operation of these networks for some special application area, the values of all energy generators and consumers must be given, so a model for each of them should be done. A very extensive bibliography can be found both on generation $[36,37,16,17]$ and load models [38, 39].

To validate the model, rather than apply it to some real-world or future scenario, a simple case study with six electrical agents has been created for testing the electrical layer, using the well known IEEE 6-bus system as initial outline. After that, a number of simulation experiments were designed, implemented and played, using successively further energy layers.

There are five classes of agents (for four energy layers) in the current model: Hub with 6 hub agents, Comm with 4 communication agents, Elec with 6 electrical agents, Gas with 4 chemical agents and Water with 3 thermal agents, mechanical agents are not used in this model. The Hub agents act as containers (like a multiplug) with outlets for electrical energy (red), chemical energy (green), thermal energy (blue) and communications (yellow). Electrical agents can be of types Slack, PV or PQ (as usual), and the other energy agents, thermal, chemical and mechanical (not used), can be of types Slack or PQ, and communication agents are all of the same type.

The first simulation experiment was to test the electrical part of the model using the same data as IEEE 6-bus system [32, p.104]. For this test, the authors compared the solutions obtained for this system (a) using Matlab with Matpower Toolbox [40], and (b) with the solution given by the model, and checking that the two were practically equal.

After that, a number of simulation experiments were made, by assigning special data to the other buses, loads, efficiencies, coupling factors, etc. Although the data did not belong to any given device, the simulations played correctly.

Recognized the inter-relationships between multiple energy carriers, efficiencies for each energy carriers differed and power and load sums were totaled at each tick (time- 


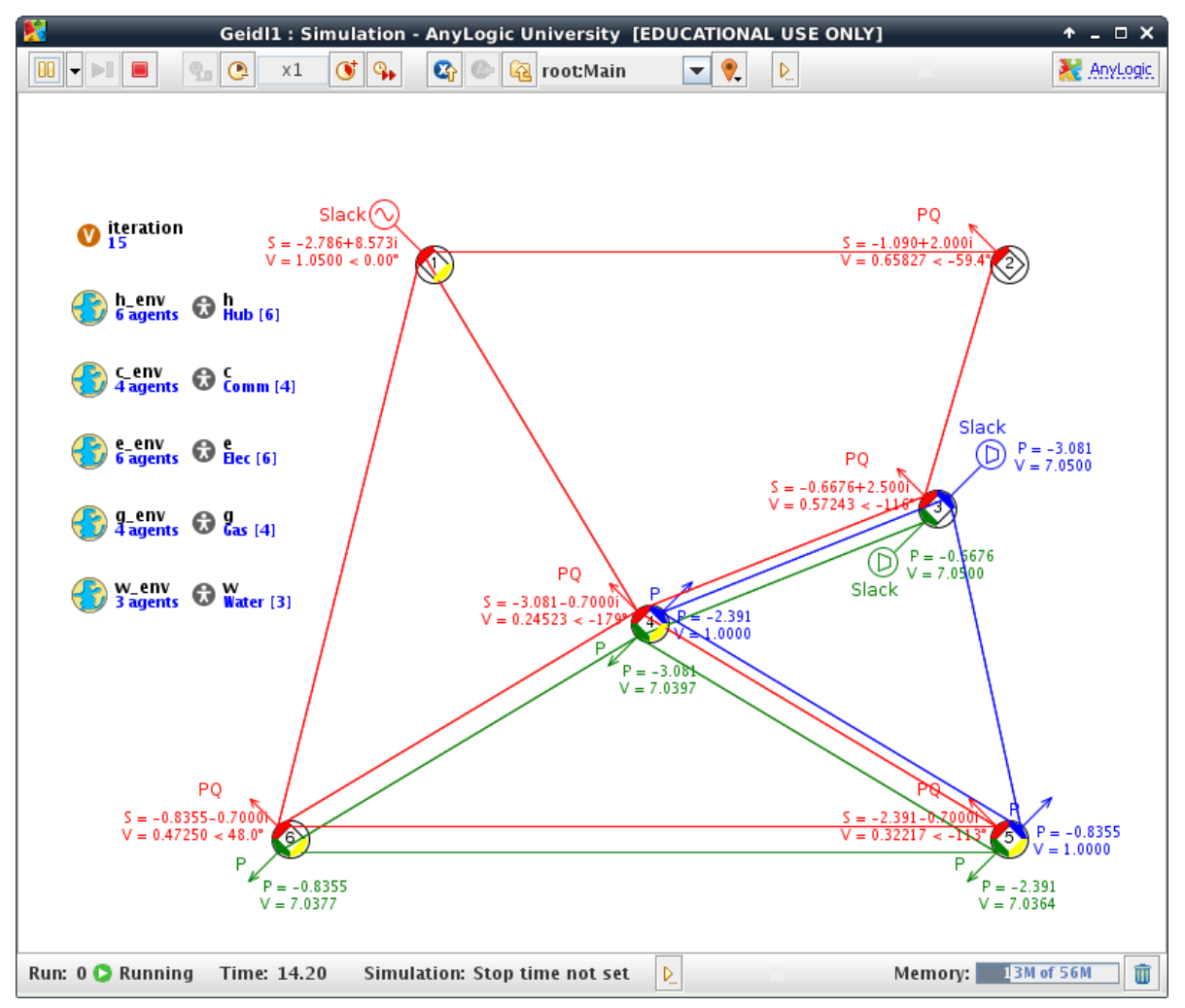

Figure 16: Multi energy carrier simulation window

step) depending on the demand from agents. The model primarily shows the dynamic rebalancing bet ween energy networks allowing for intermittency and storage. This dyamical transition is not achievable other than through bottom up, agent-led demand and supply modeling. Figure 16 shows the corresponding simulation window.

\section{Conclusion and Outlook}

In this paper, an approach for modeling generalized energy networks through an agent-based method is proposed. Considering previous works which adapted this approach to electrical networks, an extension for other energy carriers is introduced. In line with the agent-based modeling paradigm, the authors carefully chose the method to be flexible and modular. This includes modeling individual parts of the system using decentralized approaches, in which calculations are not performed in a global, but rather a local manner. In this way the model can be easily scaled or extended, by adding new entities which is possible through a modular approach.

The electrical power flow was taken as a base and simplified in order to represent flows of energy on other carriers (gas, heat, etc.). Furthermore, the concept of an energy hub is used, which functions as a conversion point located at the nodes of the network.

This standardized approach allows us to tackle the complexity of coupled energy networks. The homogeneous, consistent conceptual model, despite the high level of abstraction chosen might look very high, it allows us to consider the different carriers and their interactions, by modeling the network as a system of systems.

The model can be used to represent integrated utility infrastructures, such as systems in which not only one but different carriers are managed together by a multi-energy utility. The range of applications encompasses from small, rural or microgrid systems up to large energy infrastructures in a urban context. The model can be used to perform exploratory simulations to better get to know those systems, and further to test and develop operation management strategies.

\section{Acknowledgements}

This work was possible through the cooperation of the Complex Systems Research Centre, Cranfield University, Bedford, U.K., the European Institute for Energy Research, Karlsruhe, Germany, and the University College of Engineering, University of the Basque Country, Spain, so we would like to thank the three institutions for their support. The financial support from EPSRC for Liz Varga on project entitled 'Transforming Utilities' Conversion Points" (no. EP/J005649/1) is gratefully acknowledged. Also the authors would like to express their gratitude to the editors of ECM, and the reviewers who offered us a constructive comments and suggestions. 


\section{References}

[1] H. Merz, 3. Weltklimakonferenz (WCC-3): Genf, 31.08.2009, [Reden von Hans-Rudolf Merz als Bundespräsident], Eidgenössisches Finanzdepartement EFD, 2009.

[2] D. Energie, Energy infrastructure priorities for 2020 and beyond - A Blueprint for an integrated European energy network, ISBN 978-92-79-18877-0, 2011.

[3] I. E. Agency, Energy Technology Perspectives 2012: Pathways to a Clean Energy System, International Energy Agency, Renouf Publishing Company Limited, ISBN 9789264174887, 2012.

[4] N. Pantazis, S. Nikolidakis, D. Vergados, Energy-Efficient Routing Protocols in Wireless Sensor Networks: A Survey, Communications Surveys Tutorials, IEEE 15 (2) (2013) 551-591, ISSN 1553-877X.

[5] F. Ren, M. Zhang, D. Soetanto, X. Su, Conceptual Design of A Multi-Agent System for Interconnected Power Systems Restoration, Power Systems, IEEE Transactions on 27 (2) (2012) 732740, ISSN 0885-8950, doi:10.1109/TPWRS.2011.2177866.

[6] M. Geidl, Integrated Modeling and Optimization of Multicarrier Energy Systems, Ph.D. thesis, Swiss Federal Institute of Technology (ETH), Zurich, 2007.

[7] S. e. a. Gómez, Diffusion dynamics on multiplex networks, Physical Review Letters 110 (028701) (2013) 1-13.

[8] J. M. Gonzalez de Durana, O. Barambones, E. Kremers, L. Varga, Agent based modelling of local energy networks as tractable instances of Complex Infrastructure Systems, in: European Conference on Complex Systems ECCS-13, 2013.

[9] Final Report on the August 14, 2003 Blackout in the United States and Canada: Causes and Recommendations, Tech. Rep., U.S.-Canada Power System Outage Task Force, URL https://reports .energy .gov/BlackoutFinal-Web.pdf, 2004.

[10] E. Kremers, J. M. G. de Durana, O. Barambones, Emergent synchronisation properties of a refrigerator demand side management system, Applied Energy 101 (0) (2013) 709-717, ISSN 0306-2619, Sustainable Development of Energy, Water and Environment Systems.

[11] E. Kremers, J. G. de Durana, O. Barambones, Multi-agent modeling for the simulation of a simple smart microgrid, Energy Conversion and Management 75 (0) (2013) 643 - 650, ISSN 0196-8904, doi: http://dx.doi.org/10.1016/j.enconman.2013.07.050.

[12] M. Rylatt, R. Gammon, P. Boait, L. Varga, P. Allen, M. Savill, R. Snape, M. Lemon, B. Ardestani, V. Pakka, G. Fletcher, S. Smith, D. Fan, M. Strathern, CASCADE: An Agent Based Framework for Modeling The Dynamics of Smart Electricity Systems, Emergence: Complexity \& Organization 15 (2).

[13] K. Heussen, S. Koch, A. Ulbig, G. Andersson, Energy storage in power system operation: The power nodes modeling framework, in: Innovative Smart Grid Technologies Conference Europe (ISGT Europe), 2010 IEEE PES, 1 -8, doi: 10.1109/ISGTEUROPE.2010.5638865, 2010.

[14] J. M. González de Durana, O. Barambones, Object oriented simulation of Hybrid Renewable Energy Systems focused on Supervisor Control, in: IEEE Conference on Emerging Technologies and Factory Automation, 2009, ETFA 2009, 1-8, 2009.

[15] J. González de Durana, O. Barambones, E. Kremers, P. Viejo, Complete Agent based Simulation of Mini-Grids, in: The Ninth IASTED European Conference on Power and Engineering Systems, EuroPES 2009, vol. 681, Acta Press, Palma de Mallorca, Spain, 046-088, 2009.

[16] E. Kremers, N. Lewald, O. Barambones, J. González de Durana, An Agent-based Multi-Scale Wind Generation Model, in: The Ninth IASTED European Conference on Power and Engineering Systems, EuroPES 2009, vol. 681, Acta Press, Palma de Mallorca, Spain, 064-166, 2009.

[17] E. Kremers, P. Viejo, J. M. González de Durana, O. Barambones, A Complex Systems Modelling Approach for Decentralized Simulation of Electrical Microgrids, in: 15th IEEE International Conference on Engineering of Complex Computer Systems, Oxford, 2010.
[18] J. González de Durana, O. Barambones, E. Kremers, P. Viejo, Modelling smart grids as complex systems through the implementation of intelligent hubs, in: $7^{\text {th }}$ International Conference on Informatics in Control, Automation \& Robotics, ICINCO 2010, Conference proceedings, Funchal, Madeira - Portugal, 2010.

[19] P. D. Gallagher, NIST Framework and Roadmap for Smart Grid Interoperability Standards, NIST Special Publication 1108R2, 2012.

[20] B. Bollobás, Modern Graph Theory, Graduate texts in mathematics, Springer, Heidelberg, corrected edn., ISBN 038-7-984887, 1998.

[21] W.-K. Chen, Graph Theory and Its Engineering Applica, Advanced Series in Electrical and Computer Engineering, World Scientific, ISBN 9789810218591, 1997.

[22] S. Karnouskos, A. Izmaylova, Simulation of web service enabled smart meters in an event-based infrastructure, in: Industrial Informatics, 2009. INDIN 2009. 7th IEEE International Conference on, 125-130, 2009.

[23] H. T. Zhang, L. L. Lai, An overview on smart grid simulator, in: Power and Energy Society General Meeting, 2012 IEEE, ISSN 1944-9925, 1 -6, doi:10.1109/PESGM.2012.6345201, 2012.

[24] A. L. Barabasi, The Architecture of Complexity, Control Systems Magazine, IEEE 27 (4) (2007) 33-42, 0272-1708.

[25] P. G. Doyle, J. Snell, Laurie, Random Walks and Electric Networks URL http://math.dartmouth.edu/ doyle/docs/walks/walks.pdf.

[26] P. Wellstead, Introduction to physical system modelling, Mathematics in Science and Engineering series, Academic Press, ISBN 9780127443805, 1979 .

[27] A. Bergen, V. Vittal, Power System Analysis, Prentice Hall, New Jersey, 2000.

[28] J. Grainger, W. Stevenson, Power System Analysis, McGrawHill, 1 edn., ISBN 9780070612938, 1994.

[29] L. Varga, Transforming Critical Infrastructure, International Innovation (2013) 35-37ISSN 2041-4552.

[30] R. Frik, P. Favre-Perrod, Proposal for a multifunctional energy bus and its interlink with generation and consumption, Diploma thesis, High Voltage Laboratory, ETH, Zurich, 2004.

[31] R. Sedgewick, K. Wayne, Introduction to programming in Java - an interdisciplinary approach, Pearson/Addison Wesley, ISBN 978-0-321-49805-2, 2008

[32] A. Wood, B. Wollenberg, Power Generation, Operation and Control, John Wiley \& Sons, 1996.

[33] M. Vrahatis, G. Magoulas, V. Plagianakos, From linear to nonlinear iterative methods, Applied Numerical Mathematics 45 (1) (2003) 59-77, ISSN 0168-9274, doi:10.1016/S01689274(02)00235-0, 5th IMACS Conference on Iterative Methods in Scientific Computing 28-31 May, 2001, Heraklion, Crete (Greece).

[34] K. Schneider, D. Chassin, Y. Chen, J. Fuller, Distribution power flow for smart grid technologies, in: Power Systems Conference and Exposition, 2009. PSCE '09. IEEE/PES, 1-7, 2009.

[35] F. Milano, Power System Analysis Toolbox, URL Available: http://www. power. uwaterloo.ca/ fmilano/psat.htm, 2009

[36] J. Paatero, Computational Studies on Variable Distributed Energy Systems, Ph.D. thesis, Doctoral dissertation, Helsinki University of Technology, Espoo, Finland, 94 pages, 2009.

[37] I. Richardson, M. Thomson, D. Infield, C. Clifford, Domestic electricity use: A high-resolution energy demand model, Energy and Buildings 42 (10) (2010) 1878-1887.

[38] M. Stokes, Removing barriers to embedded generation: a fine-grained load model to support low voltage network performance analysis, Ph.D. thesis, De Montfort University, The Gateway, Leicester LE1 9BH, United Kingdom, URL http://hdl.handle.net/2086/4134, 380 pages, 2005.

[39] J. Paatero, P. Lund, A model for generating household electricity load profiles, International Journal of Energy Research 30 (5) (2006) 273-290.

[40] P. S. E. R. Center, Matpower Toolbox, URL Available: http://www . pserc. cornell. edu/matpower/, 2009. 